PROCEEDINGS OF THE

AMERICAN MATHEMATICAL SOCIETY

Volume 129, Number 10, Pages 2819-2822

S 0002-9939(01)05871-3

Article electronically published on February 22, 2001

\title{
POWER LINEAR KELLER MAPS OF DIMENSION THREE
}

\author{
CHARLES CHING-AN CHENG
}

(Communicated by Wolmer V. Vasconcelos)

\begin{abstract}
In this paper it is proved that a power linear Keller map of dimension three over a field of characteristic zero is linearly triangularizable.
\end{abstract}

Let $K$ be a field. A polynomial map $F$ in dimension $n$ over $K$ is an $n$-tuple $\left(F_{1}, F_{2}, \cdots, F_{n}\right)$ of polynomials in $K\left[X_{1}, X_{2}, \cdots, X_{n}\right]$. If $G$ is another polynomial map of the same dimension, then the composition of $F$ and $G$ is defined by

$$
F \circ G=\left(F_{1}\left(G_{1}, G_{2}, \cdots, G_{n}\right), \cdots, F_{n}\left(G_{1}, G_{2}, \cdots, G_{n}\right)\right) .
$$

The polynomial map $F$ is invertible if there exists a polynomial map $G$ such that $F \circ G$ and $G \circ F$ are both identities. It is Keller if the determinant of its Jacobian is a nonzero element in $K$, i.e., det $J F \in K^{*}$. By the chain rule for Jacobians, invertible polynomial maps are Keller maps. The famous Jacobian conjecture states that if char $K=0$, then any Keller map is invertible (see, e.g., 1 or [4).

A polynomial map $F$ is power linear if it is of the form $\left(X_{1}+A_{1}^{d_{1}}, X_{2}+A_{2}^{d_{2}}, \cdots\right.$, $\left.X_{n}+A_{n}^{d_{n}}\right)$ where $A_{i}$ is a linear form in $X_{1}, X_{2}, \cdots, X_{n}$ and $d_{i} \geq 2$ for all $i$. It is cubic linear if it is power linear where $d_{i}=3$ for all $i$. Druzkowski [2] showed that in the case char $K=0$ if cubic linear Keller maps are invertible, then the Jacobian conjecture would be true. A polynomial map is triangular if it is of the form $\left(X_{1}+p_{1}, X_{2}+p_{2}, \cdots, X_{n}+p_{n}\right)$ where $p_{i}$ is a polynomial in $K\left[X_{i+1}, X_{i+2}, \cdots, X_{n}\right]$. It is linearly triangularizable if there exists a linear invertible polynomial map $\phi$ such that $\phi \circ F \circ \phi^{-1}$ is triangular. A polynomial map is elementary if it is of the form $\left(X_{1}, \cdots, X_{i-1}, X_{i}+p, X_{i+1}, \cdots, X_{n}\right)$ where $p \in K\left[X_{1}, \cdots, X_{i-1}, \hat{X}_{i}, X_{i+1}, \cdots\right.$, $\left.X_{n}\right]$. It is tame if it can be written as a composition of invertible linear maps and elementary maps. It is not hard to see that linearly triangularizable maps are tame and tame maps are invertible. The tame generators conjecture asserts that an invertible polynomial map is tame. This is proved in dimension two by Jung [5] and van der Kulk 6]. For dimensions beyond two, it is an open problem.

In this note we prove that power linear Keller maps in dimension three over a field of characteristic zero are linearly triangularizable (hence tame and invertible) proving both the Jacobian and the tame generators conjecture in this case. (It is worth noting that if the degrees of the components of these maps are all equal to three, then the result is a special case of that of Wright [7] which states that all cubic homogeneous polynomial maps are linearly triangularizable.)

Received by the editors August 1, 1999 and, in revised form, February 2, 2000.

2000 Mathematics Subject Classification. Primary 14R15, 14R10.

Key words and phrases. Polynomial map, invertible map, linearly triangularizable map, tame, Jacobian conjecture. 
Theorem. Let $K$ be a field of characteristic zero and let $F=\left(X+A^{d_{1}}, Y+\right.$ $\left.B^{d_{2}}, Z+C^{d_{3}}\right)$ be a polynomial map over $K$ with $d_{i} \geq 2$ for all $i$ where $A, B, C$ are linear forms in $X, Y, Z$. If $\operatorname{det} J F \in K^{*}$, then $F$ is linearly triangularizable.

Proof. Let $A=a_{1} X+a_{2} Y+a_{3} Z, B=b_{1} X+b_{2} Y+b_{3} Z, C=c_{1} X+c_{2} Y+c_{3} Z$. For convenience let

$$
[x y]_{p q}=\left[\begin{array}{ll}
x_{p} & x_{q} \\
y_{p} & y_{q}
\end{array}\right]
$$

and

$$
[x y z]_{p q r}=\left[\begin{array}{lll}
x_{p} & x_{q} & x_{r} \\
y_{p} & y_{q} & y_{r} \\
z_{p} & z_{q} & z_{r}
\end{array}\right]
$$

We also let $(x y)_{p q}=\operatorname{det}[x y]_{p q}$ and $(x y z)_{p q r}=\operatorname{det}[x y z]_{p q r}$. By expanding $\operatorname{det} J F$ and collecting homogeneous components we see that $\operatorname{det} J F \in K^{*}$ (or, equivalently, $\operatorname{det} J F=1)$ is equivalent to

$$
\begin{aligned}
d_{1} a_{1} A^{d_{1}-1}+ & d_{2} b_{2} B^{d_{2}-1}+d_{3} c_{3} C^{d_{3}-1}+d_{1} d_{2}(a b)_{12} A^{d_{1}-1} B^{d_{2}-1} \\
& +d_{1} d_{3}(a c)_{13} A^{d_{1}-1} C^{d_{3}-1}+d_{2} d_{3}(b c)_{23} B^{d_{2}-1} C^{d_{3}-1} \\
& +d_{1} d_{2} d_{3}(a b c)_{123} A^{d_{1}-1} B^{d_{2}-1} C^{d_{3}-1}=0
\end{aligned}
$$

(Case 1) Suppose two of $A, B, C$ are zero. After linear conjugation by an appropriate permutation map, we may assume that $B=C=0$. (For example if $A=B=0$, then, after conjugating $F$ with $(Z, Y, X)$, we get $F_{1}=(Z, Y, X) \circ$ $F \circ(Z, Y, X)=\left(X+\bar{C}^{d_{3}}, Y, Z\right)$ where $\bar{C}(X, Y, Z)=C(Z, Y, X)$.) By $(\mathrm{J})$, we have $d_{1} a_{1} A^{d_{1}-1}=0$. Since $d_{1} \geq 2$, either $a_{1}=0$ or $A=0$. In both cases, $F$ is triangular.

(Case 2) Suppose only one of $A, B, C$ is zero. As before, after conjugating with a permutation map, we may assume that $C=0$. Suppose $A, B$ are similar, i.e., $B=r A$ for some $r \in K^{*}$, and $d_{1}=d_{2}$. Then $F_{1}=\phi \circ F \circ \phi^{-1}=\left(X+\bar{A}^{d_{1}}, Y, Z\right)$ where $\phi=\left(X, Y-r^{d_{1}} X, Z\right)$ and the result follows from (Case 1). Otherwise either $A, B$ are not similar or $d_{1} \neq d_{2}$. Now $(\mathrm{J})$ implies the following:

$$
\begin{gathered}
d_{1} a_{1} A^{d_{1}-1}+d_{2} b_{2} B^{d_{2}-1}=0 \\
d_{1} d_{2}(a b)_{12} A^{d_{1}-1} B^{d_{2}-1}=0 .
\end{gathered}
$$

Since $d_{i}>0,(1)$ implies $a_{1}=b_{2}=0$ and (2) implies $(a b)_{12}=0$. Consequently, the matrix

$$
\left(\begin{array}{ll}
a_{1} & a_{2} \\
b_{1} & b_{2}
\end{array}\right)
$$

has a row of zeros. After conjugating $F$ with $(Y, X, Z)$, we may assume that $a_{1}=$ $b_{1}=b_{2}=0$, i.e., $F$ is triangular.

(Case 3) Suppose none of $A, B, C$ are zero. After conjugating with a permutation map we may assume that $d_{1} \geq d_{2} \geq d_{3}$. There are two cases. 
(i) $\left(d_{1}-1\right) \neq\left(d_{2}-1\right)+\left(d_{3}-1\right)$. Then $(\mathrm{J})$ is equivalent to

$$
\begin{aligned}
d_{1} a_{1} A^{d_{1}-1}+d_{2} b_{2} B^{d_{2}-1}+d_{3} c_{3} C^{d_{3}-1} & =0, \\
d_{1} d_{2}(a b)_{12} A^{d_{1}-1} B^{d_{2}-1}+d_{1} d_{3}(a c)_{13} A^{d_{1}-1} C^{d_{3}-1} & \\
+d_{2} d_{3}(b c)_{23} B^{d_{2}-1} C^{d_{3}-1} & =0, \\
d_{1} d_{2} d_{3}(a b c)_{123} A^{d_{1}-1} B^{d_{2}-1} C^{d_{3}-1} & =0 .
\end{aligned}
$$

First note that at most two of $A, B, C$ are similar. For if $A, B, C$ are similar, then we may assume $d_{1}, d_{2}, d_{3}$ are distinct or else it is reduced to (Case 2) by a linear conjugation as before. Henceforth we may assume $d_{1}>d_{2}>d_{3}$. Now (J1) implies that $a_{1}=b_{2}=c_{3}=0$ and so $A=B=C=0$, contradicting the assumption.

Suppose first that only two of $A, B, C$ are similar. Then, after linear conjugation with an appropriate permutation map, we may assume that $C=r B$ for some $r \in$ $K^{*}$ and that $A, B$ are not similar. If $d_{2}=d_{3}$, then, as before, a linear conjugation reduces the problem to (Case 2). Otherwise after a linear conjugation, we may assume that $d_{3}>d_{2}$. After substituting $C=r B$ and dividing through by $B^{d_{2}-1}$ in (J2) we have

$$
\left(d_{1} d_{2}(a b)_{12}+d_{1} d_{3}(a c)_{13} r^{d_{3}-1} B^{d_{3}-d_{2}}\right) A^{d_{1}-1}=-d_{2} d_{3}(b c)_{23} r^{d_{3}-1} B^{d_{3}-1} .
$$

Since $d_{3} \geq 2, B$ divides the right side, hence the left side. Since $A$ is not similar to $B$, we have $d_{1} d_{2}(a b)_{12}+d_{1} d_{3}(a c)_{13} r^{d_{3}-1} B^{d_{3}-d_{2}}=d_{2} d_{3}(b c)_{23} r^{d_{3}-1}=0$ and so $(a b)_{12}=(a c)_{13}=(b c)_{23}=0$. Substituting $C=r B$ into (J1), we have

$$
d_{1} a_{1} A^{d_{1}-1}=-d_{2} b_{2} B^{d_{2}-1}-d_{3} c_{3} r^{d_{3}-1} B^{d_{3}-1} .
$$

Since $d_{i} \geq 2$ for all $i, B$ divides the left side. But $A, B$ are not similar, so both sides are zero. Consequently, $a_{1}=0$ and, as $d_{2} \neq d_{3}, b_{2}=c_{3}=0$. Since $C=r B$, this implies that $b_{3}=c_{2}=0$. Note that $b_{1} \neq 0$; otherwise $B=0$. So $(a b)_{12}=0$ implies $a_{2}=0$ and, similarly, $(a c)_{13}=0$ implies $a_{3}=0$. Therefore $A=0$, a contradiction.

It remains to treat the case where no two of $A, B, C$ are similar. After linear conjugation with an appropriate permutation map, we may assume $d_{1} \geq d_{2} \geq d_{3}$. From (J2), we have

$$
d_{1} d_{2}(a b)_{12} A^{d_{1}-1} B^{d_{2}-1}+d_{1} d_{3}(a c)_{13} A^{d_{1}-1} C^{d_{3}-1}=-d_{2} d_{3}(b c)_{23} B^{d_{2}-1} C^{d_{3}-1} .
$$

Since $d_{1}>1, A$ divides the right side. Since $A$ does not divide either $B$ or $C$, $(b c)_{23}=0$ and, since $A \neq 0, d_{1} d_{2}(a b)_{12} B^{d_{2}-1}+d_{1} d_{3}(a c)_{13} C^{d_{3}-1}=0$. But since $d_{2}>1$ and $B, C$ are not similar, we have $(a b)_{12}=(a c)_{13}=0$. By $(\mathrm{J} 3),(a b c)_{123}=0$ so $\operatorname{rank}[a b c]_{123}<3$. Since $A, B$ are not similar, $C=p_{1} A+p_{2} B$. Therefore, $(a c)_{13}=p_{2}(a b)_{13},(b c)_{23}=-p_{1}(a b)_{23}$. Since, by assumption, $p_{1} \neq 0$ and $p_{2} \neq 0$, we have $(a b)_{12}=(a b)_{13}=(a b)_{23}=0$. Hence the first two rows of $[a b c]_{123}$ are linearly dependent, i.e., $A$ is similar to $B$, a contradiction.

(ii) $\left(d_{1}-1\right)=\left(d_{2}-1\right)+\left(d_{3}-1\right)$. Then $(\mathrm{J})$ is equivalent to $(\mathrm{J} 3)$ and the following:

$$
\begin{aligned}
d_{2} b_{2} B^{d_{2}-1}+d_{3} c_{3} C^{d_{3}-1} & =0, \\
d_{1} a_{1} A^{d_{1}-1}+d_{2} d_{3}(b c)_{23} B^{d_{2}-1} C^{d_{3}-1} & =0, \\
d_{1} d_{2}(a b)_{12} A^{d_{1}-1} B^{d_{2}-1}+d_{1} d_{3}(a c)_{13} A^{d_{1}-1} C^{d_{3}-1} & =0 .
\end{aligned}
$$

As before if $A, B, C$ are similar, then we may assume that $d_{1}>d_{2}>d_{3}$. Therefore (K1) implies $b_{2}=c_{3}=0$; hence $b_{3}=c_{2}=0$ and consequently $(b c)_{23}=0$. Using this in (K2) we have $a_{1}=0$ and so $A=B=C=0$, a contradiction. 
Suppose only two of $A, B, C$ are similar. We may also assume as before that the powers of these similar forms are not equal. We may further assume $B, C$ are similar. Then (K1) implies $b_{2}=c_{3}=0$, (K2) implies $a_{1}=(b c)_{23}=0$, (K3) implies $(a b)_{12}=(a c)_{13}=0$ and $(\mathrm{J} 3)$ implies $(a b c)_{123}=0$.

It is easy to arrive at the same conclusion if no two of $A, B, C$ are similar. By [3] Lemma 1.2], there exists a permutation matrix $P$ such that $P[a b c]_{123} P^{-1}$ is upper triangular with 0 's on the main diagonal. Hence $F$ is linearly triangularizable.

\section{REFERENCES}

[1] H. Bass, E. Connell, and D. Wright, The Jacobian conjecture: reduction of degree and formal expansion of the inverse, Bull. Amer. Math. Soc. 7 (1982), 287-330. MR 83k:14028

[2] L. M. Dru.zkowski, An effective approach to Keller's Jacobian conjecture, Math. Ann. 264 (1983), 303-313. MR 85b:14015a

[3] L. M. Dru.zkowski, The Jacobian conjecture in case of rank or corank less than three, J. Pure Appl. Algebra 85 (1993), 233-244. MR 93m:14011

[4] A. R. P. van den Essen, Seven lectures in polynomial automorphisms, Automorphisms of Affine Spaces, Kluwer, 1995, pp. 3-39.

[5] H. W. E. Jung, Über Ganze birationale Transformationen der Ebene, J. Reine Angew Math. 184 (1942), 161-174. MR 5:74f

[6] W. van der Kulk, On polynomial rings with two variables, Nieuw archief voor Wiskunde 1 (1953), 33-41. MR 14:941f

[7] D. Wright, The Jacobian conjecture: linear triangularization for cubics in dimension three, Linear and Multilinear Algebra 34 (1993), 85-97. MR 96j:14008

Department of Mathematics and Statistics, Oakland University, Rochester, MichiGAN 48309-4401

E-mail address: cheng@oakland.edu 